\title{
Diurnal Spatial Rearrangement of Microglial Processes through the Rhythmic Expression of P2Y ${ }_{12}$ Receptors
}

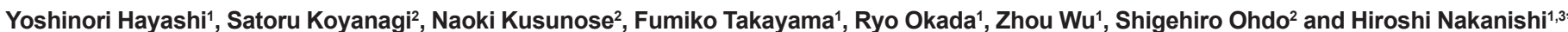

${ }^{1}$ Faculty of Dental Sciences, Department of Aging Science and Pharmacology, Kyushu University, Fukuoka, Japan

${ }^{2}$ Faculty of Pharmaceutical Sciences, Pharmaceutics, Kyushu University, Fukuoka, Japan

${ }^{3}$ Japan Science and Technology Agency, CREST, 5, Sanbancho, Chiyoda-ku, Tokyo, Japan

\begin{abstract}
Microglia plays important roles in synaptic reorganization during the postnatal developmental stage. Moreover microglia continuously surveys the functional state of the synapse and change to improve the function. This phenomenon was attributed to the fine process of extension and retraction. However, the mechanism underlying the dynamics of microglial movement and function is still unclear. We herein report that cortical microglia exhibit clock gene-regulated diurnal morphological changes. Cortical microglia extended their processes during the dark phase and retracted them during the light phase. These diurnal changes were also observed in cortical microglia from animals housed under constant darkness, but not in cortical microglia from clock-mutant mice. The mean contact ratio of the microglia-synapse interactions was significantly larger during the dark phase than the light phase. These diurnal changes in microglial morphology and microglia-synaptic interactions were significantly inhibited by the systemic administration of clopidogrel, a $P 2 Y_{12}$ receptor $\left(P 2 Y_{12} R\right)$ blocker. We further observed diurnal variation in the $P 2 Y_{12} R$ expression in cortical microglia. The reporter analyses further revealed that $P 2 Y_{12} R$ was regulated by a negative feedback loop of the clock system. These observations suggest that the microglial clock system drives the diurnal morphological changes of microglia and microglia-synapse interactions by controlling the $\mathrm{P} 2 \mathrm{Y}_{12} \mathrm{R}$ expression.
\end{abstract}

Keywords: Microglia; Microglia-synapse interaction; $\mathrm{P}_{12}$ receptor; Clopidogrel; Clock genes

\section{Introduction}

Several lines of evidence suggest that microglia continuously move their processes by extension and retraction $[1,2]$. The motile fine processes of microglia are directly contact with synaptic structures [3], implying that microglia regulate the functional state of neurotransmission in the normal brain. During the postnatal developmental stage, microglia have an important role in the regulation of synapse maturation with phagocytosis occurring through CX3CR1 signaling in the hippocampus or barrel cortex [4,5]. In addition, microglia engulfs and eliminates unnecessary synapses in a complement-dependent manner in the dorsal lateral geniculate nucleus [6]. Microglia also eliminates dendritic spines to adapt to environmental alterations in the visual cortex [7]. These observations strongly suggest that microglial processes have important roles in the fine tuning of the neural circuit in the healthy brain.

Microglial processes contact synaptic sites by their recognition of neuronal activity. A reduction of the neuronal activity in the visual cortex after an injection of tetrodotoxin into the retina resulted in a decreased frequency of contacts between microglial processes and synaptic sites [3]. On the other hand, the neuronal activity is continuously changing during the day. It is well known that the suprachiasmatic nucleus exhibits a daily oscillation in neuronal activity, which regulates the circadian rhythm [8]. Diurnal neuronal activity was also reported in the cerebral cortex $[9,10]$. Furthermore, the total net change in the dendritic spine is also regulated by the sleep-wake cycle $[11,12]$. These observations prompted us to examine the possible involvement of microglia in the diurnal changes in the neuronal synaptic activity through microglia-synapse interactions. Therefore, in the present study, we examined the possible association of the diurnal changes with the microglia-synapse interactions using a quantitative morphological approach. We herein provide the first evidence of the diurnal morphological changes in microglia and microglia-synapse interactions, which are regulated by clock gene- driven expression of $\mathrm{P} 2 \mathrm{Y}_{12}$ receptors $\left(\mathrm{P} 2 \mathrm{Y}_{12} \mathrm{R}\right)$.

\section{Materials and Methods}

\section{Animals}

All efforts were made to minimize animal suffering and to reduce the number of animals used. Eight-week-old male C57BL/6 mice and Clock-mutant (Clock/Clock) mice, which have a point mutation in exon 19 of the Clock gene (Jackson Laboratory, Bar Harbor, ME, USA), on an ICR background were purchased from Charles River Japan, Inc. (Kanagawa, Japan). The mice were housed under either normal light/dark (LD) condition (light on at 07:00 and light off at 19:00) or constant darkness (DD) in a temperature-controlled room $\left(24 \pm 1^{\circ} \mathrm{C}\right)$ with a humidity of $60 \pm 10 \%$, and were provided food and water ad libitum. They were adapted to the LD for two weeks before the experiments. Under the LD condition, the ZT0 (Zeitgeber time) was designated as lights on and ZT12 as lights off. For mice housed under the DD condition for one day, the circadian time (CT) was used instead of ZT, and CT0 was characterized as the beginning of the subjective light phase, and CT12 was defined as the beginning of the subjective dark phase. All the preparations were performed under the red light. All animals were treated in accordance with the guidelines stipulated by the animal care and use committee of Kyushu University.

*Corresponding author: Dr. Hiroshi Nakanishi, Faculty of Dental Sciences, Department of Aging Science and Pharmacology, Kyushu University, Fukuoka, 812-8582, Japan, Fax: +81 92642 6415; E-mail: nakan@dent.kyushu-u.ac.jp

Received May 20, 2013; Accepted June 19, 2013; Published June 21, 2013

Citation: Hayashi Y, Koyanagi S, Kusunose N, Takayama F, Okada R, et al. (2013) Diurnal Spatial Rearrangement of Microglial Processes through the Rhythmic Expression of P2Y ${ }_{12}$ Receptors. J Neurol Disord 2: 120. doi:10.4172/2329-6895.1000120

Copyright: @ 2013 Hayashi Y, et al. This is an open-access article distributed under the terms of the Creative Commons Attribution License, which permits unrestricted use, distribution, and reproduction in any medium, provided the original author and source are credited. 
Citation: Hayashi Y, Koyanagi S, Kusunose N, Takayama F, Okada R, et al. (2013) Diurnal Spatial Rearrangement of Microglial Processes through the Rhythmic Expression of P2Y 12 Receptors. J Neurol Disord 1: 120. doi:10.4172/2329-6895.1000120

\section{Isolation of microglia from adult animals}

Wild-type and clock-mutant mice were anesthetized and perfused transcardially with phosphate buffered saline (PBS), then the mice were decapitated at the time point of ZT2, 6, 10, 14, 18 or 22 or at CT2 or14 ( $\mathrm{n}=3$ at each time point). Brain tissue samples were cut into small pieces and single-cell suspensions were obtained by enzymatic digestion using the Neural Tissue Dissociation Kit (Papain). The tissue was further mechanically dissociated using a wide-tipped, firepolished Pasteur pipette and the suspension was applied to a $30 \mu \mathrm{m}$ cell strainer. The cells were magnetically labeled with CD11b MicroBeads. The cell suspension was loaded onto a magnetic cell sorting (MACS) column placed in the magnetic field of a MACS Separator, and the negative fraction was collected. After removing the magnetic field, the CD11b-positive cells were eluted as a positive fraction. Isolated cells were incubated with anti-mouse CD45-fluorescein (FITC) and antimouse CD11b-phycoerythrin (PE) at optimal dilutions. Stained cells were analyzed by a fluorescent activated cell sorting (FACS) (BectonDickinson) using the Cell Quest software program. The purity of microglia was calculated to be $78.24 \%$. All reagents were purchased from Miltenyi Biotec. All manipulations were terminated within $3 \mathrm{~h}$ in the present study to prevent any effect of microglial activation due to the isolation procedure.

\section{Quantitative RT-PCR analysis}

Total RNA was extracted from microglia cells using RNAiso (Takara Bio Inc., Shiga, Japan) at ZT2, 6, 10, 14, 18 or 22 or at CT2 or 14. Complementary DNA (cDNA) was prepared via reverse transcription of the total RNA using a ReverTra Ace ${ }^{\circledR}$ qPCR RT kit (Toyobo Co. Ltd., Osaka, Japan). Diluted cDNA samples were analyzed by real-time RTPCR. Real-time PCR was performed using the THUNDERBIRD ${ }^{\mathrm{TM}}$ SYBR $^{\circledR}$ qPCR Mix (Toyobo) and the 7500 Real-time PCR system (Applied Biosystems, Foster City, CA). To determine the copy numbers, we chose the $2{ }^{-\Delta C t}$ method using a calibrator as described previously [13]. The primer sequences used for the quantitative PCR of target gene $\left(\mathrm{P} 2 \mathrm{Y}_{12} \mathrm{R}\right)$ were forward: 5'-CACAGAGGGCTTTGGGAACTTA-3' and reverse: 5'TGGTCCTGCTTCTGCTGAATC-3 and for $\beta$-actin (as a control) were forward: 5'-CACACCTTCTACAATGAGCTGC-3' and reverse: 5'-CATGATCTGGGTCATCTTTTCA-3' and the resulting amplicons were $126 \mathrm{bp}$ and $109 \mathrm{bp}$, respectively. These primers were designed to flank a region that contains at least one intron.

\section{Construction of reporter and expression vectors}

To contrast the luciferase reporter vector for the $P 2 Y_{12} R$ gene $\left(P 2 Y_{12} R-L u c\right)$, a 5 -upstream fragment of the promoter region of the mouse $P 2 Y_{12} R$ gene spanning bp -944 to +51 (the number is the distance from the putative transcription starting site, +1 ; Genbank Accession \#NM_027571) was amplified by PCR, and the product was fused to the luciferase gene using a pGL3-promoter vector (Promega). The expression plasmids for clock and clock-related genes were prepared as follows: the coding regions of clock or clock-related genes were obtained by RT-PCR and used after their sequences were confirmed, as reported previously [14]. All coding regions were ligated into the pcDNA3.1 vector (Invitrogen).

\section{Luciferase reporter assay}

The NIH3T3 cells were maintained in DMEM supplemented with $5 \% \mathrm{FBS}$ at $37^{\circ} \mathrm{C}$ in a humidified $5 \% \mathrm{CO}_{2}$ atmosphere [15]. The NIH3T3 cells do not express the $P 2 Y_{12} R$ gene in NIH3T3 cells (data not shown). The day before transfection, cells were seeded $\left(1 \times 10^{5}\right.$ cells/well $)$ into 24 well plates. The next day, the cells were transfected with $100 \mathrm{ng}$ of the reporter construct and $0.5 \mu \mathrm{g}$ (total) of expression constructs using the Lipofectamine-LXT reagent (Invitrogen, Carlsbad, CA, USA) according to the manufacturer's instructions. To correct for the variations in transfection efficiency, $0.5 \mathrm{ng}$ of pGL4.74 [hRluc/ TK] vector (Promega, San Luis Obispo, CA, USA) was co-transfected into each well. The total amount of DNA per well was adjusted by adding pcDNA3.1 vector (Invitrogen). At $48 \mathrm{~h}$ post-transfection, the cells were harvested with $200 \mu \mathrm{L}$ passive lysis buffer, and $50 \mu \mathrm{L}$ of the extracts were used for firefly luciferase and Renilla luciferase assays by luminometry. The ratio of firefly luciferase activity expressed from reporter plasmids to the Renilla luciferase activity expressed from pGL4.74 [hRluc/TK] in each sample served as a measure of the normalized luciferase activity, as reported previously [14].

\section{Histology}

Mouse tissues were examined by an immunohistochemical analysis as described previously [16]. All mice were deeply anesthetized with sodium pentobarbital $(50 \mathrm{mg} / \mathrm{kg}$ i.p.) and perfused transcardially with $0.1 \mathrm{M}$ phosphate buffer ( $\mathrm{PB} ; \mathrm{pH}$ 7.4), followed by $4 \%$ paraformaldehyde (PFA) in $0.1 \mathrm{M} \mathrm{PB}$ at ZT2, ZT14, CT2 and CT14 ( $\mathrm{n}=3$ animals at each time point) for immunohistochemistry. Transverse cortical sections (free floating, $50 \mu \mathrm{m}$ thick) were prepared using a vibratome (VT1000S; Leica, Nussloch, Germany). The floating sections were incubated with a mixture of $1.0 \%$ BSA in PBS containing $0.05 \%$ sodium azide $1 \mathrm{~h}$ at room temperature, then the sections were stained with an antibody against Ibal (1:10,000; Wako) for $7 \mathrm{~d}$ at $4^{\circ} \mathrm{C}$ and with Cy 3 conjugated donkey anti-rabbit IgG (1:400, Jackson Immunoresearch) for $3 \mathrm{~h}$ at $4^{\circ} \mathrm{C}$. The sections were then washed with PBS and mounted in the Vectashield anti-fading medium (Vector laboratories, Peterborough, UK) and examined with a confocal laser-scanning microscope (CLSM, LSM510MET, Carl Zeiss, Jena, Germany). The images of microglia were captured from layer $2 / 3$ in the primary somatosensory cortex.

\section{Intracellular labeling and subsequent Ibal staining}

The cortical segments perfused with $4 \%$ PFA were sectioned into $100 \mu \mathrm{m}$ thick samples by a vibratome (VT1000S; Leica, Nussloch, Germany) ( $n=24-26$ slices from three animals). The sections were incubated with YOYO-1 (1:10,000; Molecular Probes) for 3 min to visualize the cell body. A glass electrode filled with $4 \%$ lucifer yellow was applied to the YOYO-1-positive cell bodies under a microscope equipped with a $40 \times$ water immersion objective by using a laser with a wavelength of $488 \mathrm{~nm}$. When the dendrite apparently changed brightness following the insertion of the glass electrode into the cell body, current was injected (up to $-10 \mathrm{nA}$ ) in the current clamp mode to further inject the lucifer yellow, as was previously described for a similar protocol [17]. After the injection of the lucifer yellow, the sections were incubated with $4 \%$ PFA for $24 \mathrm{~h}$ at $4^{\circ} \mathrm{C}$. Next, the sections were further stained with an anti-Ibal antibody as described above. The images were captured by a CLSM at wavelengths of $477 \mathrm{~nm}$ and $543 \mathrm{~nm}$.

\section{Quantitative morphological analyses of microglia and den- dritic spines}

All images were processed using the Image J software program (National Institutes of Health, Bethesda, MD, USA). The acquisition of 3D images was carried out by CLSM with $63 \times$ objective fields for single staining with Ibal and with $100 \times$ objective fields for counter-staining with Iba1. Stacks of 70-90 serial optical sections were obtained from 
Citation: Hayashi Y, Koyanagi S, Kusunose N, Takayama F, Okada R, et al. (2013) Diurnal Spatial Rearrangement of Microglial Processes through the Rhythmic Expression of $\mathrm{P}_{2} \mathrm{Y}_{12}$ Receptors. J Neurol Disord 1: 120. doi:10.4172/2329-6895.1000120

Page 3 of 7

each cerebral cortex (22-27 dendrites from 10-15 cells from three animals). The morphological analyses of microglia were performed on Z-projections of confocal images. The cell number of microglia was counted for Z-projection images with a thickness of $50 \mu \mathrm{m}$ within $0.01 \mathrm{~mm}^{2}$. Microglial processes were traced and then reconstructed as single microglial images using the Simple Neurite Tracer software program plug-in. Semi-automated tracing of dendrites was performed for the 3D image data [18], which were used to calculate the total length of microglial processes and the branch number. The topological skeletonized images of single microglia were converted from a binarized image of single microglia using the skeletonize function. The spine density was determined by manually identifying the spines and using 3D images to measure the dendrite length with the Simple Neurite Tracer plug-in. The number of dendritic spines in close apposition with microglial processes or enwrapped by microglial processes was counted. The number of dendritic spines contacted with microglial processes was normalized to the total number of dendritic spines counted and represented as the contact ratio. 18-20 microglial cells were analyzed in each animal. The 3D illustrations of microglia and spines were made using the Reconstruct 1.1 software program (http://synapses.clm.utexas.edu).

\section{Immunoblotting}

The samples were separated by electrophoresis on 10\% SDSpolyacrylamide gels ( $\mathrm{n}=3$ independent experiments). Proteins on SDS gels were transferred electrophoretically to nitrocellulose membranes. The membranes were washed with PBS and incubated at $4^{\circ} \mathrm{C}$ overnight under gentle agitation with each primary antibody: anti-P2 ${ }_{12} \mathrm{R}$ (1:1,000; Novus Biologicals) and anti- $\beta$-actin $(1: 1,000$; Santa Cruz). After washing, the membranes were incubated with horseradish peroxidase (HRP)-labeled anti-goat (1:1,000; R\&D Systems) or anti-rabbit (1:1,000; GE Healthcare) antibodies for $1 \mathrm{~h}$ at room temperature. Subsequently, the membrane-bound, HRP-labeled antibodies were detected using an enhanced chemiluminescence detection system (ECK lit; GE Healthcare) with an image analyzer (LAS-4010; Fuji Photo Film).

\section{Drug administration}

Clopidogrel (25 mg/kg, LKT Laboratories, St. Paul, MN), a selective $\mathrm{P} 2 \mathrm{Y}_{12} \mathrm{R}$ inhibitor, was systematically administered three times every $3 \mathrm{~h}$ from ZT10-18 and CT10-18. Then, specimens for the histological experiments were prepared at ZT2 and CT2. In some experiments, the mice were decapitated at ZT14 and CT14 after either saline or clopidogrel administration at ZT10 and CT10.

\section{Statistical analyses}

All data are shown as the means \pm SEM. The statistical analyses were performed using two-tailed unpaired Student's $t$-tests, a oneway analysis of variance (ANOVA) with a post-hoc Tukey's test or a two-way ANOVA with repeated measurements using the GraphPad Prism Software package (GraphPad Software Inc., San Diego, CA, USA). Unless otherwise indicated, data met the assumptions of equal variances. Differences were considered to be significant at $\mathrm{p}<0.05$.

\section{Results}

\section{Diurnal morphological change in cortical microglia}

The quantitative morphological analyses of cortical microglia in the young adult $\mathrm{C} 57 \mathrm{BL} / 6$ mice were performed using the skeletonized images reconstructed from Z-stack images of Ibal-immunostained microglia (Figures 1A-1D). Diurnal variation in spatial area and structural complexity were observed in cortical microglia (Figures $1 \mathrm{E}-1 \mathrm{H})$. The mean total length of processes (Figure 1I) and the mean number of branch points (Figure 1J) of cortical microglia

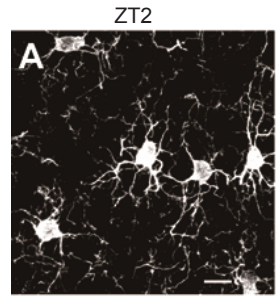

E

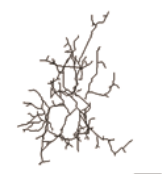

I

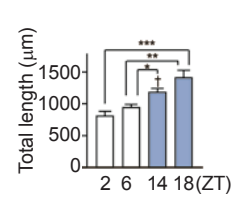

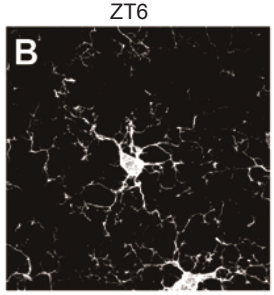

$\mathbf{F}$
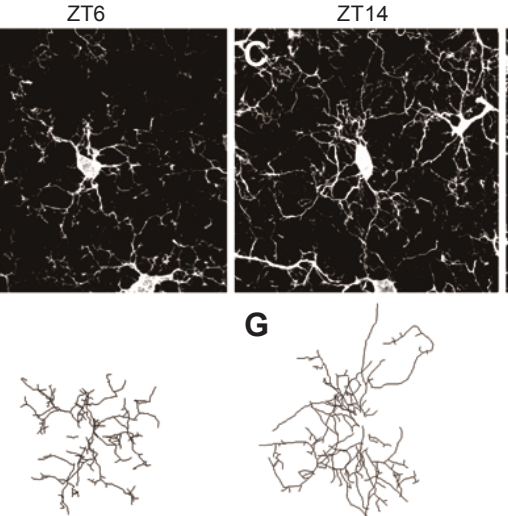

G
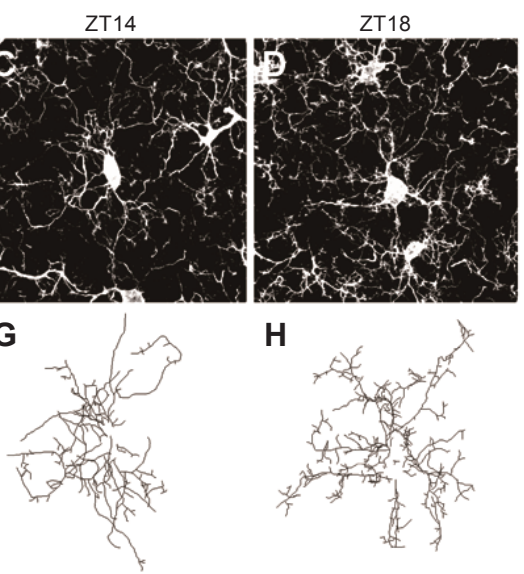

H

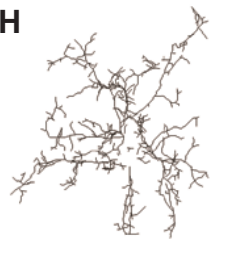

$\mathbf{J}$
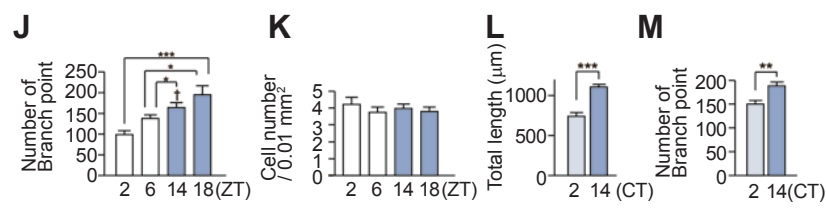

Figure 1: The circadian variations in the spatial area and structural complexity of cortical microglia.

A-D: CLSM stack images of cortical microglia from C57BL/6 mice at ZT2 (A), ZT6 (B), ZT14 (C) and ZT18 (D), E-H: The topological skeletonized images from reconstructed 2D mages of cortical microglia at ZT2 (E), ZT6 (F), ZT14 (G) and ZT18 (H). Scale bars, $10 \mu \mathrm{m}$. I-K: The total length of processes $(\mathrm{I})$, number of branch points $(\mathrm{J})$, and the number of cortical microglia $(K)$ from mice housed under the LD condition, $L$, M: The total length of processes $(L)$ and number of branch points (M) of cortical microglia in the mice housed under the DD condition.

The data are the means \pm S.E.M. For animals $=3, \mathrm{n}=24-30$ sections, each; ${ }^{*}, P<0.05 ;{ }^{* *}, P<0.01 ;{ }^{* * *}, P<0.001 ; \dagger, P<0.05$ compared with $\mathrm{ZT2}$ (one-way ANOVA) or CT2 (unpaired $t$-test). The number of microglia was normalized to an area of $0.01 \mathrm{~mm}^{2}$. 
Citation: Hayashi Y, Koyanagi S, Kusunose N, Takayama F, Okada R, et al. (2013) Diurnal Spatial Rearrangement of Microglial Processes through the Rhythmic Expression of $\mathrm{P}_{2} \mathrm{Y}_{12}$ Receptors. J Neurol Disord 1: 120. doi:10.4172/2329-6895.1000120

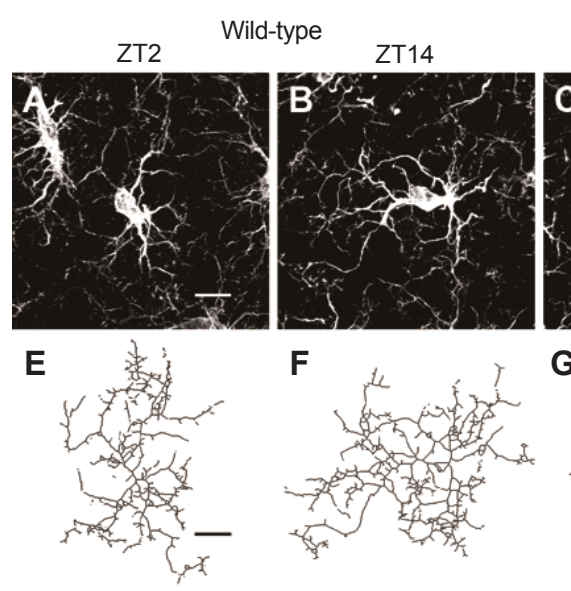

I

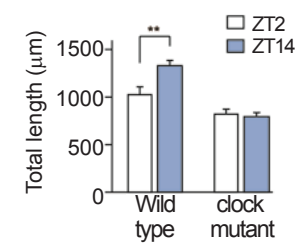

$\mathbf{J}$

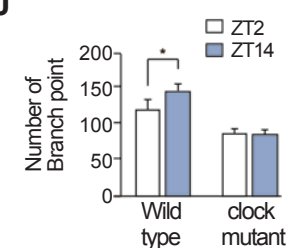

Clock-mutant

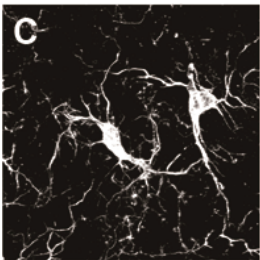

G
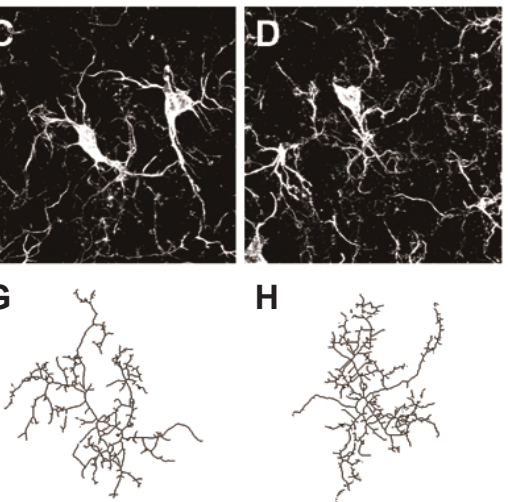

H

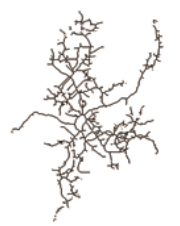

K

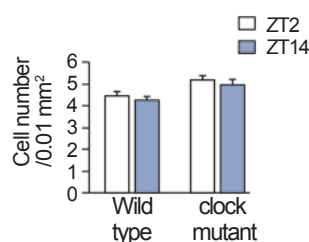

Figure 2: The deficits in the circadian variation of the spatial area and the structural complexity of cortical microglia from clock-mutant mice, A-D: CLSM stack images of cortical microglia at ZT2 (A) and ZT14 (B) from wild-type mice and ZT2 (C) and ZT14 (D) from clock-mutant mice, E-H: The topological skeletonized reconstructed images from the 2D images of cortical microglia at ZT2 (E) and ZT14 (F) from wild-type mice and ZT2 (G) and ZT14 (H) from clock-mutant mice. Scale bars, $10 \mu \mathrm{m}$. I, J: The clock mutation led to a total loss of the alterations in the process length (I) and branch points (J) of cortical microglia. The data are the means \pm S.E.M. (animals=3, $n=27-29$ sections each).

The asterisks indicate statistically significant differences between values $\left({ }^{*}, P<0.05 ;{ }^{*}, P<0.01\right.$; one-way ANOVA). K: The number of cortical microglia within a $0.01 \mathrm{~mm}^{2}$ area was not significantly different at each time point (animals=3, $\mathrm{n}=27-29$ sections each).

were significantly larger during the dark phase (Zeitgeber time; ZT14-18) than during the light phase (ZT2-6) under the light/ dark (LD) condition. However, the number of microglial cells was not significantly different (Figure $1 \mathrm{~K}$ ). Circadian variations in the spatial area and structural complexity of cortical microglia were also observed even in the mice housed under the constant darkness (DD) condition (Figure $1 \mathrm{~L}$ and $1 \mathrm{M}$ ), indicating that the intrinsic clock system generates the diurnal morphological changes. To directly examine the involvement of the circadian clock system on the diurnal morphological changes in cortical microglia, we utilized clock-mutant (Clock/Clock) mice, which exhibit disrupted circadian gene expression because of a point mutation in exon 19 of the clock gene [19]. Microglia in the somatosensory cortex of wild-type ICR mice also exhibited the diurnal morphological change of microglia (Figures 2A, 2B, 2E, 2F, 2I and 2J). In contrast, there was no diurnal morphological change of cortical microglia in clock-mutant mice (Figure 2C, 2D, 2G, 2H-2J). The number of cortical microglia was not changed at the time point examined (Figure $2 \mathrm{~K}$ ). These results clearly indicate that the circadian clock system drives the diurnal morphological changes in cortical microglia.

\section{Regulation of microglia-synapse interactions by P2Y12R}

$\mathrm{P} 2 \mathrm{Y}_{12}$ receptors $\left(\mathrm{P} 2 \mathrm{Y}_{12} \mathrm{R}\right)$, which are Gi/o-coupled ATP receptors expressed exclusively on microglia in the brain [20], regulate microglial process extension [21]. The loss of $\mathrm{P}_{2} \mathrm{Y}_{12} \mathrm{R}$ expression accompanies microglial transformation from a highly ramified to amoeboid state following neuronal injury [21]. Therefore, the possible involvement of $\mathrm{P} 2 \mathrm{Y}_{12} \mathrm{R}$ in the diurnal variation of the microglial morphological complexity was next examined using clopidogrel, a P2 $\mathrm{Y}_{12} \mathrm{R}$ blocker that can cross the blood-brain barrier after systemic administration [22].
The extension of microglial processes during the dark phase (ZT14) was significantly inhibited by the systemic treatment with clopidogrel (Figures 3A-3C). Lucifer yellow, combined with subsequent Iba1staining showed that the microglial processes frequently made contact with dendritic spines (Figure 3D). The three-dimensional (3D) reconstructed images showed that microglial processes wrapped (Figures 3E-3H) and apposed (Figures 3I-3K) the dendritic spines. The mean contact ratio of microglia and dendritic spines was significantly higher at ZT14 $(4.72 \pm 0.35 \%)$ than at ZT2 $(3.07 \pm 0.54 \%)$ (Figure 3I), and correlated with the microglial complexity (Figures $1 \mathrm{I}$ and $1 \mathrm{~J}$ ). The method used in this study may underestimate the contact ratio of microglia and dendritic spines, because microglial processes are highly motile and make only a brief contact with dendritic spines [3]. Furthermore, the increase in dendritic spine density at ZT14 $(0.85$ $\pm 0.03 / \mu \mathrm{m})$ compared with ZT2 $(0.66 \pm 0.03 / \mu \mathrm{m})$ was similar to the previously reported [11]. More interestingly, systemic administration of clopidogrel suppressed the contact ratio at ZT14 (Figure 3L; $3.05 \pm$ $0.33 \%)$ and preserved the dark phase-like dendritic spine density even at ZT2 $(0.84 \pm 0.02 / \mu \mathrm{m})$. These observations suggest that $\mathrm{P}_{2} \mathrm{Y}_{12} \mathrm{R}$ plays a key role in microglial interactions with synaptic structures.

\section{Clock gene-regulated expression of $P 2 Y_{12} R$ in cortical microglia}

To examine whether cortical microglia exhibit clock gene expression, which would regulate $P 2 Y_{12} R$ gene expression, microglia were isolated from the adult mouse cerebral cortex by a magnetic cell sorting method using CD11b microbeads at $4 \mathrm{~h}$-intervals over a $24 \mathrm{~h}$ period. Microglia was found to exhibit a circadian oscillation of $P 2 Y_{12} R$ transcripts with a peak at ZT14 (Figures 4A and 4B). Furthermore, a rhythmic pattern of $P 2 Y_{12} R$ was also observed in microglia isolated 

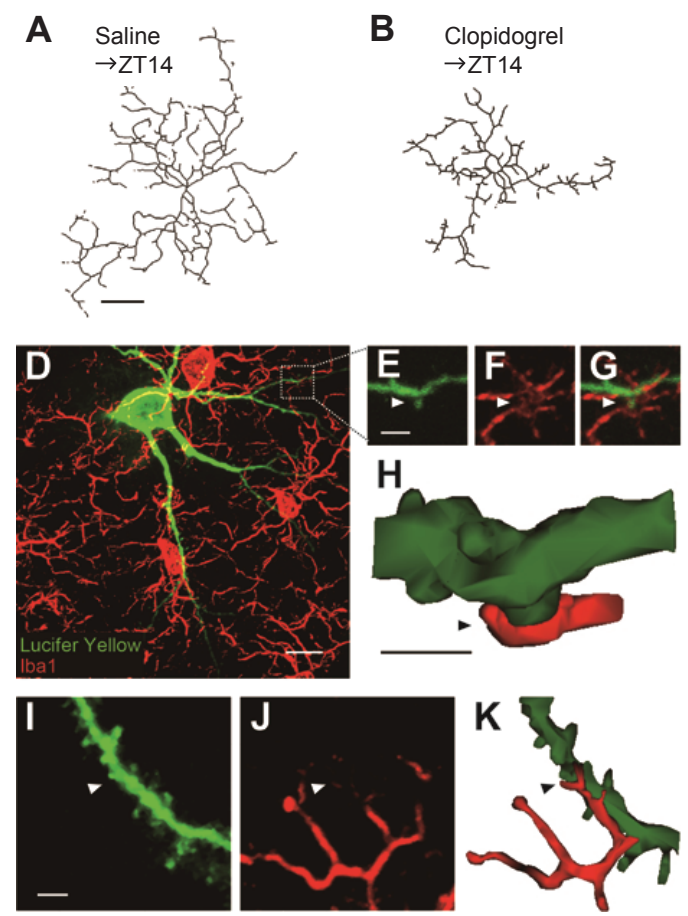

C
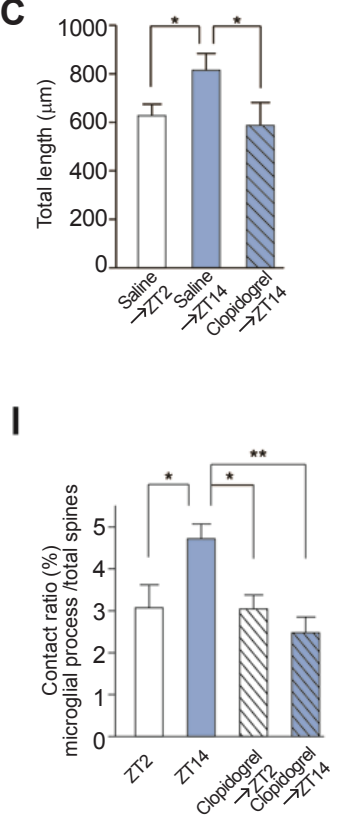

Figure 3: The inhibitory effects of clopidogrel on the circadian variation in the microglial morphology and microglia-synapse interactions, A, B: Inhibition of the time-of-day microglial process extension was induced by treatment with clopidogrel. The topological skeletonized images of cortical microglia at ZT14 after saline (A) or clopidogrel (B) administration. C: The total length of cortical microglial processes after clopidogrel treatment. The data are the means \pm S.E.M. (animals $=3$, $\mathrm{n}=24-26$ sections each; * $P<0.05$; one-way ANOVA). D: CLSM Z-stack images of lucifer yellow-injected cortical neurons (green) and microglia (red), E-H: Magnified images of dendrite $(E)$, microglia $(F)$, merged images $(G)$ and a 3D reconstruction $(H)$ of the dotted square in (D). Arrowhead: enhancement of the dendritic spines with microglial processes. I-K: The apposition images of dendritic spines (I), microglial processes $(\mathrm{J})$ and the 3D reconstruction (K). Scale bar, $10 \mu \mathrm{m}$ for $(A, D)$ and $2 \mu \mathrm{m}$ for $(E-K)$. L: The contact rate of microglial processes and spines.

The data are the means \pm S.E.M. ( $n=829-881$ spines; ${ }^{*}, P<0.05 ;{ }^{* *}, P<0.01$; one-way ANOVA).

under the DD conditions (Figure 4C). In addition, the diurnal variation in the expression of $P 2 Y_{12} R$ occurred in a clock-dependent manner (Figures $4 \mathrm{~B}$ and $4 \mathrm{C}$ ). The protein level of $\mathrm{P} 2 \mathrm{Y}_{12} \mathrm{R}$ in cortical microglia was also increased at ZT14 compared to ZT2 (Figure 4D).

The E-box site (CACGTG), the target of the circadian clock effectors BMAL1 and CLOCK, is located 382 bp upstream of $P 2 Y_{12} R$. Therefore, the relationship between the clock genes and $P 2 Y_{12} R$ was further analyzed using a luciferase reporter assay system targeting this region (Figure 4E). CLOCK/BMAL1 proteins enhanced the transcriptional activity of $P 2 Y_{12} R$ - Luc that contained the E-box, whereas CLOCK $\Delta 19$ / BMAL1 did not (Figure 4F). In addition, the CLOCK/BMAL1mediated transactivation of $P 2 Y_{12} R-L u c$ was significantly repressed by the addition of PER2 or CRY1 (Figure 4G). These observations indicate that the diurnal changes in the $P 2 Y_{12} R$ gene expression are regulated by the molecular clock system, consisting of a negative feedback loop with the CLOCK/BMAL1 complex binding to E-boxes in its promoter region.

\section{Discussion}

The major finding of this study is that cortical microglia exhibit diurnal morphological changes, which are regulated by the clock gene-driven diurnal expression of $\mathrm{P}_{2} \mathrm{Y}_{12} \mathrm{R}$. During the dark phase, microglia extends their processes resulting from an increased level of $\mathrm{P} 2 \mathrm{Y}_{12} \mathrm{R}$ expression. During the light phase, the decrease in the $\mathrm{P} 2 \mathrm{Y}_{12} \mathrm{R}$ level results in the partial retraction of microglial processes. Furthermore, this diurnal change in the microglial morphology influences the ratio of microglia-synapse interactions. It is though those microglias extend their processes specifically toward highly active synapses that release ATP and glutamate, because a reduction in the neuronal activity leads to the suppression of contact between microglial processes and dendritic spines [3]. Furthermore, sensory deprivation started the retraction of microglial process over 6 hours. Both ATP and glutamate increase the morphological complexity in microglia, such as the extension of their process and elevation of the number of branch points [23]. Glutamate itself also induces the release of ATP from microglia [23] and astrocytes [24]. ATP induces the ramification [25] and sprouting of new processes from preexisting microglial processes [26]. Consistent with these observations, apyrase, an ATP hydrolyzing enzyme, decreases the total number of branch points [23]. In our study, clopidogrel, a $\mathrm{P} 2 \mathrm{Y}_{12} \mathrm{R}$ inhibitor, suppressed the circadian microglial process extension. Besides the circadian oscillation of $\mathrm{P}_{2} \mathrm{Y}_{12} \mathrm{R}$ gene expression, extracellular ATP concentration secreted from neurons and astrocytes was also known to exhibit circadian oscillation [27,28]. Therefore, the circadian oscillation of the extracellular ATP concentration should be also considered as a causative factor for diurnal morphological variation of cortical microglia. These observations strongly suggest that ATP regulates both the length and structural complexity of microglial processes through $\mathrm{P}_{2} \mathrm{Y}_{12} \mathrm{R}$. Further studies are still needed to clarify the involvement of endogenous ATP on the process extension of microglia, and would be facilitated by the development of a bloodbrain barrier permeable ATP hydrolyzing enzyme.

Although clopidogrel is used clinically as an anti-platelet agent, clopidogrel or its metabolite(s) can cross the blood-brain barrier [29]. Furthermore, effects of clopidogrel on spinal microglia were previously observed after systemic administration [22]. The expression 
A

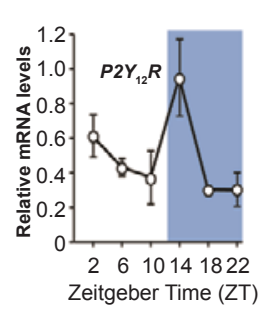

E

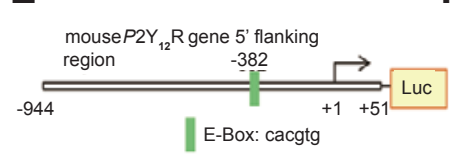

B

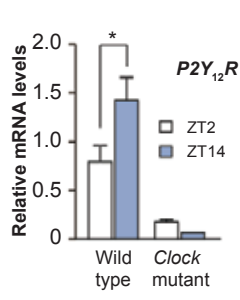

D ${ }_{\text {ZT2 ZT14 }}$

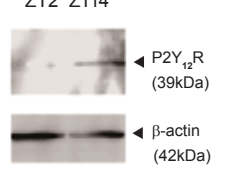

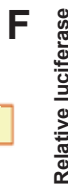

G

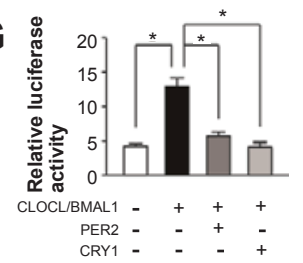

Figure 4: The possible association between the circadian changes in the microglial morphology and the P2Y12R gene expression, $\mathrm{A}$ : The circadian oscillation of the expression of the $P 2 Y_{12} R$ gene in cortical microglia in mice housed under the LD condition. The data are the means $\pm S$. $E$.M. ( $n=3$, each: $P=0.0003$; oneway ANOVA). B, C: The relative mean mRNA levels of the $P 2 Y_{12} R$ gene in microglia isolated from wild-type and clock-mutant mouse brains at $Z T 2$ and 14 (B) or CT2 and $14(\mathrm{C})$ from mice housed under the LD or DD condition. The data are the means \pm S.E.M. (n=3, each: *, $P<0.05$; one-way ANOVA). D: A P2Y12R immunoblot from microglia isolated from the cortex of adult mice at ZT2 and ZT14, E: The promoter region of the $P 2 Y{ }_{12} R$ gene was fused to a luciferase reporter gene $\left(P 2 Y_{12} R\right.$-Luc) and transfected into NIH3T3 cells, F, G: P2Y12R-Luc can be activated by CLOCK/BMAL1, but not CLOCK $19 / B M A L 1$ proteins (F) and is repressed by PER2 and CRY1 $(\mathrm{G})$.

The data are the means \pm S.E.M. $\left(n=3\right.$, each: ${ }^{* *}, P<0.01$; one-way ANOVA)

of $\mathrm{P} 2 \mathrm{Y}_{12} \mathrm{R}$ is limited to microglia, and the receptors are not expressed on neurons or astrocytes $[20,21,30]$. Therefore, it is considered that the systemic administration of clopidogrel can specifically block the $\mathrm{P}_{2} \mathrm{Y}_{12} \mathrm{R}$ expressed on microglia. The concentration of clopidogrel used in our study is extremely high compared to the clinical dose. The usual clinical dose of clopidogrel is one $75 \mathrm{mg}$ tablet per day. This concentration is equivalent to approximately $1 \mathrm{mg} / \mathrm{kg}$ in an adult human with a body weight of $60 \mathrm{~kg}$. In the present study, we administrated $25 \mathrm{mg} / \mathrm{kg}$ of clopidogrel three times a day. Therefore, it is unlikely that the usual clinical dose of clopidogrel would inhibit the microglia-synapse interactions through a blockade of the $\mathrm{P} 2 \mathrm{Y}_{12} \mathrm{R}$ expressed on microglia.

During the phagocytic elimination of dead cells in the dentate gyrus, microglia formed a ball-and chain structure on their terminal processes [31]. Furthermore, the synaptic structure is phagocytosed by microglial processes during the postnatal developmental stage $[4,6]$ and is involved in the adaptation to new environmental situations [7]. In this study, we found that the mean density of dendritic spines of the cortical neurons was significantly larger during the dark phase (ZT14) than the light phase (ZT2). Furthermore, the mean density of dendritic spines during the light phase was significantly increased following systemic administration of clopidogrel. Therefore, we expected that the increased microglia-synapse interactions during the dark phase were necessary for the phagocytic elimination of dendritic spines. However, we could not detect any structural changes in the microglial processes associated with phagocytosis (e.g. phagocytic cups) in the somatosensory cortex. Besides the phagocytic elimination of dendritic spines, extracellular proteolysis is also involved in the turnover of dendritic spines [32,33]. Microglia-derived proteases may eliminate dendritic spines of highly active synapses by the proteolysis of the extracellular matrix, resulting in the subsequent low synaptic activity during the light phase.

\section{Conclusion}

We have herein provided evidence of a novel system wherein the microglial process dynamics are regulated by the intrinsic clock system. The intrinsic microglial molecular clock drives the expression of $\mathrm{P}_{2} \mathrm{Y}_{12} \mathrm{R}$ during the dark phase to extend their processes and increase contacts with dendritic spines, which may, in turn, decrease the density of dendritic spines for the subsequent light phase. It has been proposed that dysfunction of the clock system is one of the risk factors for the psychiatric diseases. In addition, abnormal microglia has a significant association with neurological disorders [33,34]. Therefore, the microglial clock may provide valuable targets for the development of novel therapeutic agent for the neurological disorders, and further research on this topic will aid in understanding the function and dysfunction of the brain.

\section{Disclosure}

The author reports no conflicts of interest associated with this work.

\section{References}

1. Nimmerjahn A, Kirchhoff F, Helmchen F (2005) Resting microglial cells are highly dynamic surveillants of brain parenchyma in vivo. Science 308: 13141318.

2. Davalos D, Grutzendler J, Yang G, Kim JV, Zuo Y, et al. (2005) ATP mediates rapid microglial response to local brain injury in vivo. Nat Neurosci 8: 752 758.

3. Wake H, Moorhouse AJ, Jinno S, Kohsaka S, Nabekura J (2009) Resting microglia directly monitor the functional state of synapses in vivo and determine the fate of ischemic terminals. J Neurosci 29: 3974-3980.

4. Paolicelli RC, Bolasco G, Pagani F, Maggi L, Scianni M, et al. (2011) Synaptic pruning by microglia is necessary for normal brain development. Science 333: $1456-1458$

5. Hoshiko M, Arnoux I, Avignone E, Yamamoto N, Audinat E (2012) Deficiency of the microglial receptor CX3CR1 impairs postnatal functional development of thalamocortical synapses in the barrel cortex. J Neurosci 32: 15106-15111.

6. Schafer DP, Lehrman EK, Kautzman AG, Koyama R, Mardinly AR, et al. (2012) Microglia sculpt postnatal neural circuits in an activity and complementdependent manner. Neuron 74: 691-705.

7. Tremblay MĖ, Lowery RL, Majewska AK (2010) Microglial interactions with synapses are modulated by visual experience. PLoS Biol 8: e1000527. 
Citation: Hayashi Y, Koyanagi S, Kusunose N, Takayama F, Okada R, et al. (2013) Diurnal Spatial Rearrangement of Microglial Processes through the Rhythmic Expression of P2Y 12 Receptors. J Neurol Disord 1: 120. doi:10.4172/2329-6895.1000120

8. Meredith AL, Wiler SW, Miller BH, Takahashi JS, Fodor AA, et al. (2006) BK calcium-activated potassium channels regulate circadian behavioral rhythms and pacemaker output. Nat Neurosci 9: 1041-1049.

9. Liu ZW, Faraguna U, Cirelli C, Tononi G, Gao XB (2010) Direct evidence for wake-related increases and sleep-related decreases in synaptic strength in rodent cortex. J Neurosci 30: 8671-8675.

10. Vyazovskiy VV, Cirelli C, Pfister-Genskow M, Faraguna U, Tononi G (2008) Molecular and electrophysiological evidence for net synaptic potentiation in wake and depression in sleep. Nat Neurosci 11: 200-208.

11. Maret S, Faraguna U, Nelson AB, Cirelli C, Tononi G (2011) Sleep and waking modulate spine turnover in the adolescent mouse cortex. Nat Neurosci 14: 1418-1420.

12. Yang G, Gan WB (2012) Sleep contributes to dendritic spine formation and elimination in the developing mouse somatosensory cortex. Dev Neurobiol 72: 1391-1398

13. Ingham DJ, Beer S, Money S, Hansen G (2001) Quantitative real-time PCR assay for determining transgene copy number in transformed plants. Biotechniques 31: 132-134, 136-40.

14. Koyanagi S, Hamdan AM, Horiguchi M, Kusunose N, Okamoto A, et al (2011) cAMP-response element (CRE)-mediated transcription by activating transcription factor-4 (ATF4) is essential for circadian expression of the Period2 gene. J Biol Chem 286: 32416-32423.

15. Cavadini G, Petrzilka S, Kohler P, Jud C, Tobler I, et al. (2007) TNF-alpha suppresses the expression of clock genes by interfering with E-box-mediated transcription. Proc Natl Acad Sci U S A 104: 12843-12848.

16. Hayashi Y, Kawaji K, Sun L, Zhang X, Koyano K, et al. (2011) Microglia $\mathrm{Ca}(2+)$-activated $\mathrm{K}(+)$ channels are possible molecular targets for the analgesic effects of S-ketamine on neuropathic pain. J Neurosci 31: 1737017382.

17. Elston GN, Oga T, Fujita I (2009) Spinogenesis and pruning scales across functional hierarchies. J Neurosci 29: 3271-3275.

18. Cardona A, Saalfeld S, Arganda I, Pereanu W, Schindelin J, et al. (2010) Identifying neuronal lineages of Drosophila by sequence analysis of axon tracts. J Neurosci 30: 7538-7553.

19. Gekakis N, Staknis D, Nguyen HB, Davis FC, Wilsbacher LD, et al. (1998) Role of the CLOCK protein in the mammalian circadian mechanism. Science 280: 1564-1569

20. Sasaki Y, Hoshi M, Akazawa C, Nakamura Y, Tsuzuki H, et al. (2003) Selective expression of Gi/o-coupled ATP receptor P2Y12 in microglia in rat brain. Glia 44: 242-250.

21. Haynes SE, Hollopeter G, Yang G, Kurpius D, Dailey ME, et al. (2006) The
P2Y12 receptor regulates microglial activation by extracellular nucleotides. Nat Neurosci 9: 1512-1519.

22. Tozaki-Saitoh H, Tsuda M, Miyata H, Ueda K, Kohsaka S, et al. (2008) P2Y12 receptors in spinal microglia are required for neuropathic pain after periphera nerve injury. J Neurosci 28: 4949-4956.

23. Fontainhas AM, Wang M, Liang KJ, Chen S, Mettu P, et al. (2011) Microglia morphology and dynamic behavior is regulated by ionotropic glutamatergic and GABAergic neurotransmission. PLoS One 6: e15973.

24. Zhang JM, Wang HK, Ye CQ, Ge W, Chen Y, et al. (2003) ATP released by astrocytes mediates glutamatergic activity-dependent heterosynaptic suppression. Neuron 40: 971-982.

25. Wollmer MA, Lucius R, Wilms H, Held-Feindt J, Sievers J, et al. (2001) ATP and adenosine induce ramification of microglia in vitro. J Neuroimmunol 115 $19-27$

26. Xiang Z, Chen M, Ping J, Dunn P, Lv J, et al. (2006) Microglial morphology and its transformation after challenge by extracellular ATP in vitro. J Neurosci Res 83: 91-101.

27. Womac AD, Burkeen JF, Neuendorff N, Earnest DJ, Zoran MJ (2009) Circadian rhythms of extracellular ATP accumulation in suprachiasmatic nucleus cells and cultured astrocytes. Eur J Neurosci 30: 869-876.

28. Marpegan L, Swanstrom AE, Chung K, Simon T, Haydon PG, et al. (2011) Circadian regulation of ATP release in astrocytes. J Neurosci 31: 8342-8350.

29. Herbert JM, Frehel D, Vallee E, Kieffer G, Gouy D, et al. (1993) Clopidogrel, a Novel Antiplatelet and Antithrombotic Agent. Cardiovascular Drug Reviews 11: $180-198$.

30. Hollopeter G, Jantzen HM, Vincent D, Li G, England L, et al. (2001) Identification of the platelet ADP receptor targeted by antithrombotic drugs. Nature 409: 202-207.

31. Sierra A, Encinas JM, Deudero JJ, Chancey JH, Enikolopov G, et al. (2010) Microglia shape adult hippocampal neurogenesis through apoptosis-coupled phagocytosis. Cell Stem Cell 7: 483-495

32. Wang XB, Bozdagi O, Nikitczuk JS, Zhai ZW, Zhou Q, et al. (2008) Extracellular proteolysis by matrix metalloproteinase- 9 drives dendritic spine enlargement and long-term potentiation coordinately. Proc Natl Acad Sci U S A 105: 19520-19525.

33. Mataga N, Mizuguchi Y, Hensch TK (2004) Experience-dependent pruning of dendritic spines in visual cortex by tissue plasminogen activator. Neuron 44: 1031-1041.

34. Abbott LF, Nelson SB (2000) Synaptic plasticity: taming the beast. Nat Neurosci 3: 1178-1183.
This article was originally published in a special issue, Microglia and Synaptic Reorganization handled by Editor. Dr. Hiroshi Nakanishi, Kyushu

University, Japan 